\title{
THE PRESENT STATUS OF THE MUNICH DUST COUNTER EXPERIMENT ON BOARD OF THE HITEN SPACECRAFT
}

E.Igenbergs, A.Hüdepoh1 [1], K.Uesug1, T.Hayash1 [2], H.Svedhem [3], H.Iglseder [4], G.Koller [5], A.Glasmachers [6], E.Grün [7], G.Schwehm [3], H.MizutanI, T.Yamamoto, A. Fujimura, N.Ish11, H.Arak1 [2], K.Yamakosh1 [8], K. Nogami [9]

[1] Lehrstuhl für Raumfahrttechnik, Technische Universität München, Richard Vagner Str. 18, 8000 München 2, FRG

[2] Institute of Space and Astronautical Science, J

[3] European Space Research and Technology Centre of ESA, NL

[4] Center for Applied Space Technology and Microgravity, Universität Bremen, FRG

[5] Lehrstuhl für ProzeBrechner, Technische Universität München, FRG

[6] Mikroelektronik-Zentrum, Ruhr-Universität Bochum, FRG

[7] Max-Planck-Institut für Kernphysik, Heidelberg, FRG

[8] Institute for Cosmic Ray Research, University of Tokyo, J

[9] Dep. of Physics, Dokkyo Dniversity School of Medicine, J.

ABSTRACT. The Munich Dust Counter (MDG) is a scientific experiment on board the MUSES-A mission of Japan measuring cosmic dust. The satellite HITEN of this mission has been launched on January 24th, 1990 from Kagoshima Space Center. Here the present status of the MDC experiment is summarized. The number of dust particles measured so far is presented together with first and preliminary results of flux calculations and spatial as well as directional distributions of cosmic dust particles measured until July 25, 1990. A clear evidence of particles coming from the inner solar system (beta-meteoroids) already has been found. These are compared to particles coming from the apex direction.

\section{Introduction}

The Munich Dust Counter (MDC) determines mass and velocity of cosmic dust particles by measuring the charges generated by the high velocity impacts of these particles on a gold target. It is installed on the Japanese HITEN spacecraft launched on January 24, 1990 in a high elliptic orbit around the earth. The details of the instrument and of the mission of the spacecraft are discussed by Igenbergs et al. (1990) and Uesugi et al. (1990).

A.C. Levasseur-Regourd and H. Hasegawa (eds.), Origin and Evolution of Interplanetary Dust, 15-20. (1991 Kluwer Academic Publishers, Printed in Japan. 
Generally the MDC can measure particles with masses between $10^{-15}$ to $10^{-7}$ grams and velocities between 1 and $70 \mathrm{~km} / \mathrm{s}$. As it is mounted on the perimeter of the spinning spacecraft it scans the whole ecliptic plane during one revolution of the satelifte, the spin axis direction of which is maintained perpendicular to the ecliptic plane. The effective sensor area is $100 \mathrm{~cm}^{2}$, the fleld of view is $148^{\circ}$.

After the instrument switch-on on January 31, 1990, and initial tests and optimizations the nominal operation of the MDC started on March 3 , 1990. Since then the MDC is measuring continuously. So far no quantitative evaluation of the data, the determination of dust particle mass or velocity, has been carried out.

\section{Signal Types}

Included in this paper are data gathered up to July 25, 1990. In these first 144 days of nominal operation since March a large number of signals have been measured of which about 67 are supposed to be particle impacts. The signals measured so far can be arranged into eight types which are shown in $\mathrm{fig}$. 1 .

Every measurement made by the MDC consists of two curves, one for the negative impact charges, labeled EC (electron channel), and one for the positive impact charges, labeled IC (Ion channel). In the case of a particle impact one or both curves should rise, with risetimes between some $\mu \mathrm{s}$ and $100 \mu \mathrm{s}$, see Igenbergs et al. (1990).

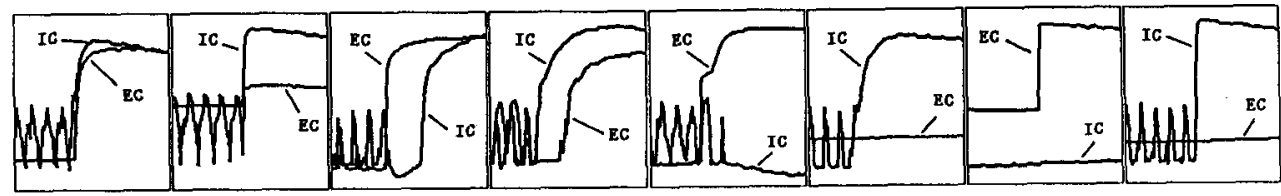
T.1
T.2
T. 3
T. 4
T. 5
T. 6
T. 7
T.8

Figure 1. Signal types encountered by the MDC experiment

Signals of type 1 to 4 show the typical shape expected for dust particle impacts. While type 1 is just perfect, the type 2 signals have some noise on the EC. Type 3 signals show a remarkable delay on the IC, while type 4 show a delay on the EC. Type 5 signals show only a signal on the EC, type 6 only a signal on the IC. All these effects are due to certain impact locations inside the sensor and could be verified during the calibration of the instrument. Type 7 signals have a very fast burst on the EC, type 8 a very fast burst on the IC. These signals are supposed to be noise and not due to particle impacts. Not included in fig. 1 is the noise generated by the sun shining into the detector opening, which has a very flat rise and has to be deleted by the MDC microprocessor system about once every revolution of the satellite.

The following graphs and diagrams have been made by using all signals of types 1 to 6 . 


\section{Particle Flux}

The MDC is measuring cosmic dust particles in its normal operational state since March 3, 1990. The diagram in fig. 2 shows the particle flux rate in impacts per day over the time in days from launch on January 24 , 1990. During the initial tests dedicated to the adaptation of the experiment software to the noise generated by the sun already four particle impacts have been measured. The 67 dust impacts measured in about 140 days of nominal operation give an average of 0.48 impacts per day, indicated as dotted Iine in fig. 2, which corresponds to a flux rate of $5.5 \cdot 10^{-4} \mathrm{~m}^{-2} \mathrm{~s}^{-1}$ for particle masses between $10^{-15}$ and $10^{-7}$ grams.

The flux diagram may suggest events like "groups" or "swarms" found with previous dust experiments, Hoffmann et al. (1975), Fechtig et al. (1979), but there is not yet enough data sampled for a good statistical treatment. Therefore no further evaluation is presented here.

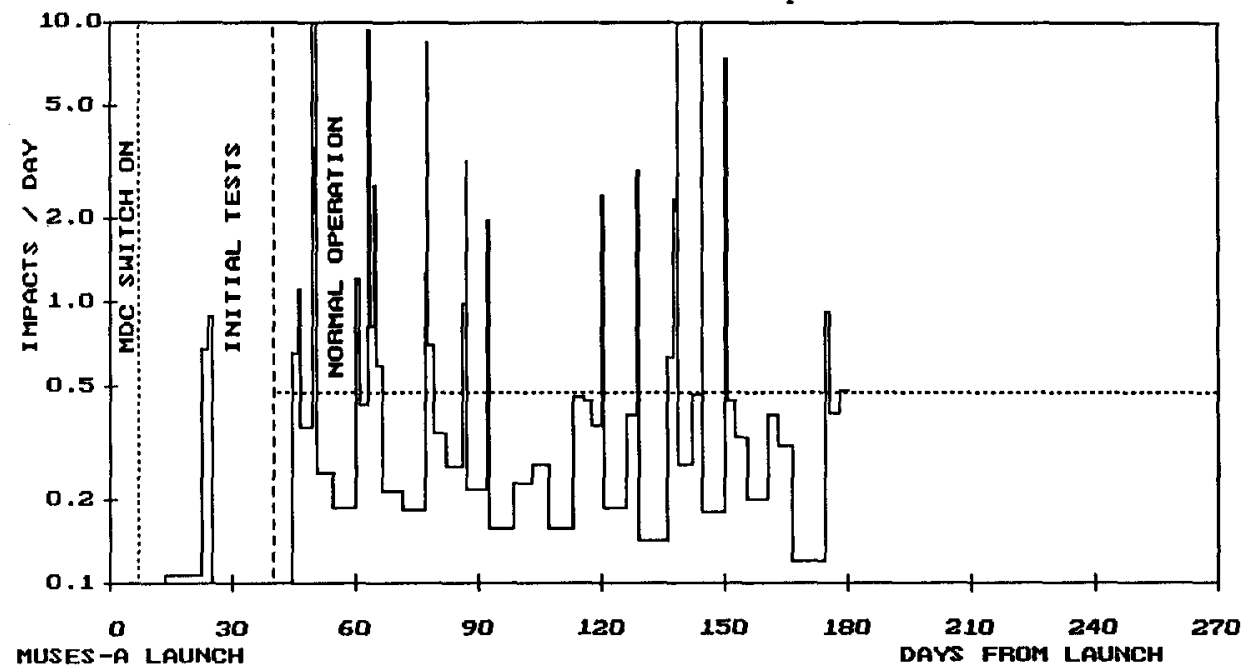

Figure 2. Cosmic dust flux as measured by the MDC in impacts per day

\section{Spatial Distribution of Dust Particles}

The orbit of the HITEN spacecraft covers nearly the whole sphere of influence of the earth with perigees ranging from some $1000 \mathrm{~km}$ to about $100000 \mathrm{~km}$ and apogees between $300000 \mathrm{~km}$ and more than 1 million $\mathrm{km}$.

Figure 3 shows a plot of the orbits of HITEN and the moon with dust particle measurements indicated. The orbits are plotted in a rotating coordinate frame with the line connecting the sun and the earth fixed and pointing left. The lines marking every impact indicate the direction in which the MDC was pointed at the time of measurement.

Here the spatial distribution of the dust particles seems to be quite uniform, but it has to be kept in mind that due to the high-eccentric orbit of the satellite the MDC is for a much longer time far away from earth than it is near the earth. A better approach will be to define 
appropriate regions of distances from earth and calculate the flux rates accordingly. This will be done when enough data are gathered.

This plot shows also that more particles have been measured from the apex direction and sun direction than from the anti-apex direction. This will be discussed in detail in the next section.

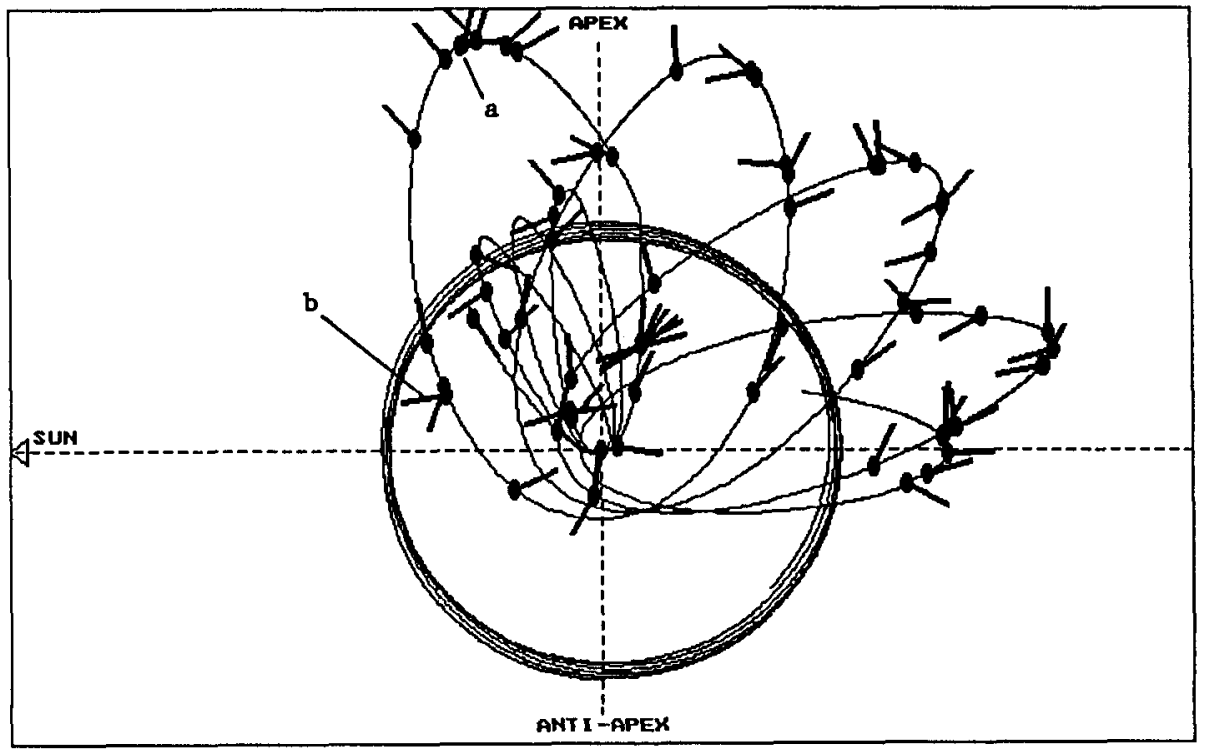

Figure 3. Orbit plot of the HITEN satellite. Positions of dust impact measurements are indicated, the lines show the MDC orientation. A typical apex-particle measurement is marked with "a", a typical beta-meteoroid measurement is marked with "b", see section 5 .

\section{Directional Distribution of Dust Particles}

As the MDC is scanning the ecliptic plane about every three seconds, it has the possibility to gather information about the particle flight directions, Of course, due to the field of view of $148^{\circ}$ of the MDC, both in ecliptic and in north/south directions, the particle flight direction determination for a single measurement is not accurate at all. But by putting together many measurements a quite clear directional distribution can be obtained. The diagram shown in fig. 4 gives the number of impacts measured for different directions. This graph was obtained by calculating the impact probability according to the projection of the sensor area to the incident angles spread over $148^{\circ}$ and adding up all measurements gathered so far.

With about 67 particle measurements displayed in the graph of fig. 4 no good statistics are possible, therefore all results have to be treated as preliminary. Up to now it seems that about 508 of the particles come roughly from the apex direction, with the peak offset outwards away from the sun. But, this effect may be due to noise which periodically 
occurs at about this angular position and which is currently under detailed investigation. Another 278 come from the sun direction, with the peak offset towards the anti-apex direction. The minimum is quite clear in the ant1-apex direction with less than 58 of all measurements.

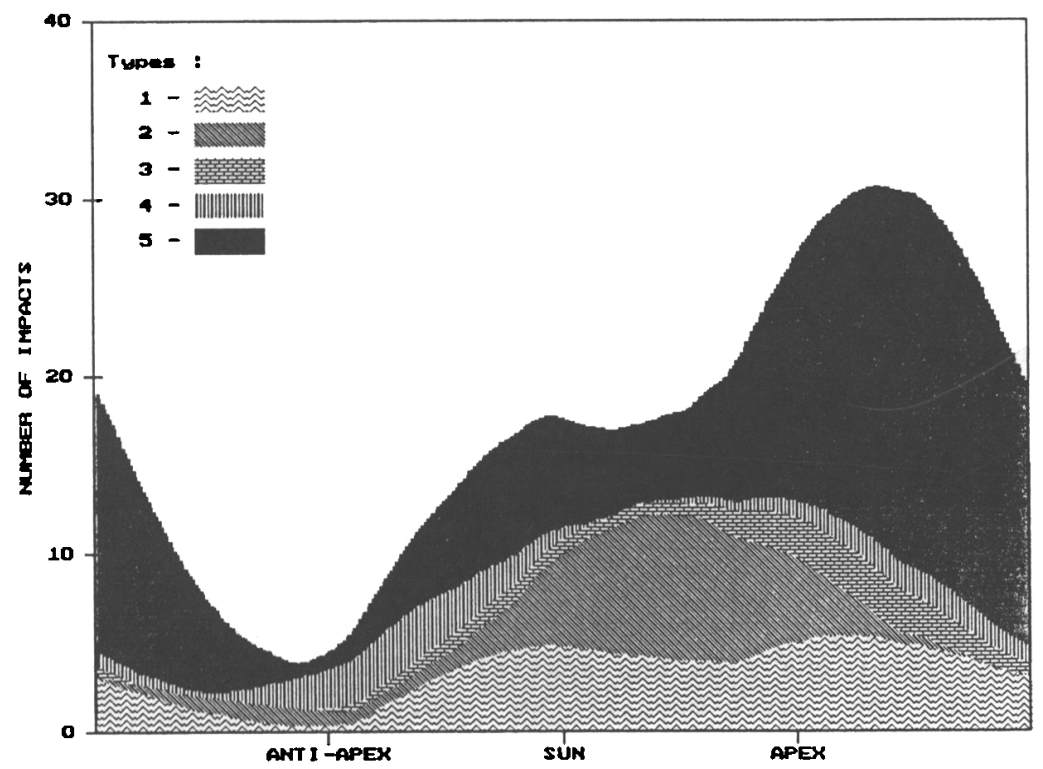

Figure 4. Spin phase angle distribution of dust particles measured by the MDC.

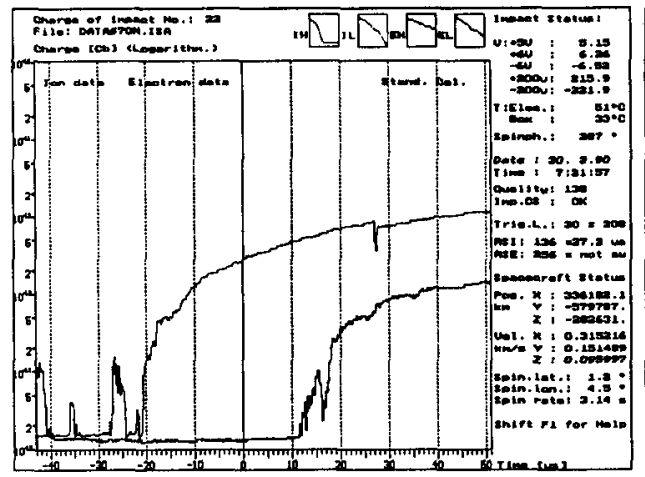

Figure 5a. Typical impact signal of an apex-particle, position of this measurement is marked in fig. 3 with " $a$ ".

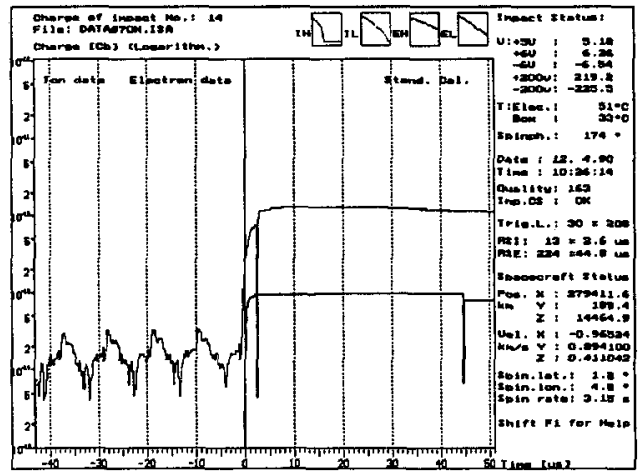

Figure 5b. Typical impact signal of a beta-meteoroid, position of this measurement is marked in fig. 3 with "b".

By comparing the signals of a particle coming from the inner solar system (beta-meteoroid), Zook and Berg (1975), with a signal of an apexparticle, Grün and Zook (1980), the typical features of these two 
classes can be distinguished. Figure 5a shows the typical impact signal of an apex-particle, marked in fig. 3 with " $a$ ". The long risetimes of the signals give a particle velocity in the order of $3 \mathrm{~km} / \mathrm{s}$. In $f i g$. $5 \mathrm{~b}$ the typical signal generated by the impact of a beta-meteorold is shown, this measurement is marked in $\mathrm{fig}$. 3 with "b". The short risetimes of the curves indicate a particle velocity in the order of $50 \mathrm{~km} / \mathrm{s}$. As the satellite velocities were quite low, these values can be taken as an indication for the velocities of the particles relative to the earth system. The masses of the particles are considered to be in the order of $10^{-10} \mathrm{~g}$ in the case of the apex-particle and $10^{-14} \mathrm{~g}$ in the case of the beta-meteorold for these specific measurements, which may not be typical for the two populations.

\section{Conclusions}

The cosmic dust experiment Munich Dust Counter is successfully measuring cosmic dust particles in the mass range between $10^{-15}$ and $10^{-7}$ grams and in the velocity range between 1 and $70 \mathrm{~km} / \mathrm{s}$ on board of the Japanese spacecraft HITEN. From March 3 until July 25, 1990, about 67 dust particles have been measured in total, which do not yet allow a good statistical treatment of the data.

By a preliminary classification of the measurements made so far informations about the flux and spatial distribution of the cosmic dust particles in the earth-moon-system could be gained. The angular distribution of the dust measured by the MDC already seems to confirm the presence of apex-particles and beta-meteoroids.

The primary phase of the mission of the HITEN satellite will end in March 1991, then a thorough data evaluation on the basis of hopefully more than 150 measurements will be possible.

\section{References}

Fechtig H., Grün E., Morfill G. (1979) 'Micrometeorolds within Ten Earth Radii', Planet. Space Sci., 27, 511-531.

Grün E., Zook H.A. (1980) 'Dynamics of Micrometeoroids', in Halliday I. and McIntosh B.A. (eds.), Solid Particles in the Solar System, Reidel Pub. Co., London, 293-298.

Hoffmann H.-J., Fechtig H., Grün E., Kissel J.(1975) 'Temporal Fluctuations and Anisotropy of the Micrometeorold Flux in the Earth-Moon System Measured by HEOS 2', Planet. Space Sci., 23, 985-991.

Igenbergs E., et al. (1990) 'The Munich Dust Counter - A Cosmic Dust Experiment on Board of the MUSES-A Mission of Japan', Proc. of the IAU Colloquium No. 126 "Origin and Evolution of Interplanetary Dust", Kyoto.

Uesugi K., Matsuo H., Kawaguchi J. Hayashi T. (1990) 'Japanese first Double Lunar Swingby Mission HITEN', Proc. of the 4lst Congress of the IAF, Dresden.

Zook H.A., Berg O.E. (1975) 'A Source for Hyperbolic Cosmic Dust Particles', Planet. Space Sci., 23, 183-203. 